\title{
Deep Learning for Real-time R-peak Prediction
}

\author{
Peishan Zhou \\ School of Engineering and Built Environment \\ Gold Coast campus, Griffith University \\ Gold Coast, Australia \\ peishan.zhou@griffithuni.edu.au
}

\author{
Belinda Schwerin \\ School of Engineering and Built Environment \\ Gold Coast campus, Griffith University \\ Gold Coast, Australia \\ b.schwerin@griffith.edu.au
}

\author{
Brent Lauder \\ School of Engineering and Built Environment \\ Gold Coast campus, Griffith University \\ Gold Coast, 4222, Australia \\ brent.lauder@griffithuni.edu.au
}

\begin{abstract}
In this work, we propose a novel algorithm to achieve real-time R-peak prediction during ECG signal recording. More specifically, from the current frame of ECG signal, we aim to predict how far into the future the next R-peak will occur, taking into account information about the variability in the intervals between beats seen in previous frames of the ECG signal. Currently there has been little work in this area. However, the real-time prediction of the next beat has important research significance, including timing of artificial heart pumps and integration into the cardiopulmonary support heart (CPS-heart), as well as narrowing the search range of $R$-peaks to assist $R$ peak detection. This paper proposes use of an integrated network using one-dimensional convolution network (1D CNN) with long short-term memory (LSTM) network. The deep learning model we have proposed is shown to effectively predict $R$-peaks with results of preliminary studies achieving a prediction accuracy of $90.61 \%$.

Index Terms-ECG, R-peak prediction, Real-time, Deep learning
\end{abstract}

\section{INTRODUCTION}

Cardiovascular diseases (CVDs) are the number 1 cause of death globally. An estimated 17,700,000 people die each year from CVDs, which accounts for $31 \%$ of all deaths worldwide according to World Health Organization (WHO) records [1]. Electrocardiogram (ECG) signals, which represent the electrical activity of the heart, is an efficient and widely used tool for detection and diagnosis of cardiovascular diseases [2].

The cardiac cycle of the ECG signal includes three main characteristic wavelets: the $\mathrm{P}$ wave, QRS complex wave, and $\mathrm{T}$ wave. Of these, the QRS complex is a particularly important component for heart health analysis and arrhythmia diagnosis. Moreover, accurate $\mathrm{R}$ peak detection is the benchmark for arrhythmia diagnosis and Heart Rate Variability (HRV) analysis [3]. HRV has been shown to be a reliable indicator of heart health, is correlated with mortality, and

\author{
Stephen So \\ School of Engineering and Built Environment \\ Gold Coast campus, Griffith University \\ Gold Coast, 4222, Australia \\ s.so@griffith.edu.au
}

has been found to indicate potential future health problems, including concussions, as well as autonomic dysfunction, such as anxiety, depression, asthma, and sudden infant death. It is also viewed as a strong indicator for the onset of myocardial infarctions. Therefore, the importance of R-peak detection is self-evident.

In recent years, with the development of a new branch of machine learning called deep learning (DL), we have witnessed their application to image processing, signal processing and speech processing, with excellent results. In addition, DL models have shown better performance and adaptability for time-series signals, such as bio-signals. The traditional Neural Network (NN) model needs to use the features extracted from the segmented heartbeat as input. DL models have recently been applied to the automatic extraction of features such as R-peaks, which are required for critical wave detection and classification. Some studies have proved through experiments that for ECG data, the hidden information automatically extracted by DL technology is more informative than the manual extraction by medical experts [4].

Convolution neural networks $(\mathrm{CNN})$ is a type of deep neural network which performs well in image processing tasks, and shows encouraging performance in physiological signal processing. Xiang et al. [5] used a simple two-layer 1DCNN network for QRS complex detection, reporting a positive prediction rate of $99.91 \%$. Moreover, effective features and visual information have been extracted from a noisy signal with a deep 1D-CNN network [6], [7]. Note, however, that although the 1D-CNN layer can adapt to different types of QRS complexes, it cannot directly predict the timing information [5], so the dense layer is used for time processing of CNN features.

As an extension of the recurrent neural networks (RNN), the long short-term memory (LSTM) network developed by Hochreiter and Schmidhuber [8] can capture the long-term dependence of data in the time dimension. For chaotic time 
series such as ECG signals, the LSTM network is indicated to provide excellent performance. The LSTM layer can also offer the further advantage of eliminating high-frequency noise in the ECG signal [9].

A recently developed approach combined 1D-CNN and LSTM into a stack model. This has been used for speech recognition [10], QRS complex detection [9] and arrhythmia diagnosis [11], [12], all achieved promising results. This combined model was shown to provide better results than using LSTM or CNN alone [10]. This is because LSTM considers the temporal correlation between ECG samples, that is, highly important information will be retained and propagated backward, while irrelevant information will be forgotten and discarded. It can continue to extract time information from the features extracted by 1D-CNN, so it shows better performance.

This paper proposes a novel method of R-peak prediction based on a one-dimensional CNN and a LSTM ensemble network. This method can realize the real-time prediction of each future R-peak from previous ECG signal frames, effectively predicting the variance in the beat to beat interval of the signal. The proposed model used for R-peak prediction is trained from sequences of ECG signals, using an ensemble of CNN and LSTM. This allows us to take advantage of the benefits of both architectures. The 1D-CNN is fast in calculation, capturing visual patterns to obtain low-dimensional features. In addition, it can filter noise so it is not sensitive to the quality of ECG signals [13]. LSTM is then used to learn the temporal dynamics in the features extracted by $1 \mathrm{D}-\mathrm{CNN}$ feature.

Development of R-peak prediction methods such as that proposed in this work, offer considerable significance. Their applications include the reduction of false R-peak detections in R-peak detection algorithms for improved HRV calculation, and realisation of improved time of beats in a cardiopulmonary support heart (CPS-heart). Moreover, the results of this research can be realized for real-time prediction in remote realtime monitoring systems or devices, such as Holter monitors and Apple Watches.

The rest of this article is organized as follows. Section II gives a brief overview of the database used. In Section III, data processing methods are introduced in detail, including a proposed data segmentation and custom shuffle schemes, and a label computation method. The structure of the CNN-LSTM integrated model is also proposed, and the design principle of the model is discussed in detail. Section IV introduces the experiment, then reports and discusses the experimental results. Finally, Section V discusses conclusions and future work.

\section{DATASETS}

In this work, ECG records provided by the MIT-BIH arrhythmia database are utilized. The database contains a total of 48 excerpts of two-channel records taken from 47 subjects. These records were chosen at random from more than 4000 24-hour Holter records collected by the Beth Israel Hospital Arrhythmia Laboratory from 1975 to 1979 , and the relevant time information and beat category information annotations have been completed by the MIT and Beth Israel Hospital. The ECG signal in the database is sampled at 360 hertz with 11-bit resolution over a $10 \mathrm{mV}$ range. Each data is a time series of approximately 30 minutes, and consists of 650000 sample points [14].

The database has been widely used in QRS complex detection research and is a popular choice for algorithm development. The 48 records of the MIT-BIH arrhythmia database are divided into two subsets, of which 23 records numbered between 100 and 125 are regarded as the first subset. The remaining 25 records numbered between 200 and 234 are the second subset, which contains patients of clinical significance, such as ventricular, junctional, and supraventricular arrhythmias.

In this research, we used 23 records from the first subset of the MIT-BIH arrhythmia database as the dataset. The training process recommended by the AAMI standard allows up to 5 minutes of a signal frame to be used from each patient's record [15]. However, an inter-patient testing scheme is utilized, which means that the training and test sets will not share any of the same records. The training and test sets are randomly divided at a ratio of $90 \% / 10 \%$. Additionally, in order to monitor the training process and prevent the model from overfitting, a $10 \%$ validation set is randomly divided from the training set records. Therefore, in the 23 records, 18 ECG records are used for training, two records and three records are used for validation and test respectively.

\section{METHOD}

The proposed method includes three stages in order to train the model used for R-peak prediction using the training data set described above. These are: the Data preparation stage; the label computation stage; and the CNN-LSTM model training stage. Each of these stages are described as follows.

\section{A. Data Preparation}

1) Noise Reduction: In order to keep the raw format of the ECG signal as much as possible, only the most basic filtering is performed through the FIR filter. No further noise reduction preprocessing (such as additional noise or artifact suppression techniques) is applied. Since the FIR filter causes a delay of (M-1)/2 samples where (M) is the number of taps for the filter, a compensator is applied to offset the delay. Fig. 1 shows the raw and filtered ECG segment in red and green, respectively, for record 100 .

2) Segmentation Scheme: The length of the ECG record in the MIT-BIH database is about 30 minutes. After filtering, every recording is segmented with a fixed 1000 sample wide sliding window, corresponding to approximately 3 seconds. In addition, the update length of the sliding window is set to 25 samples. This is long enough to simplify training, helping to reduce training time and improve the ergodicity of model training, while being short enough to ensure sufficient frames of training data are available to result in an effective model. These segments are saved in a 2D matrix for later model training, that is, as the input of the $1 \mathrm{D}-\mathrm{CNN}$ layer. Fig. 2 shows a detailed explanation of this segmentation method: 

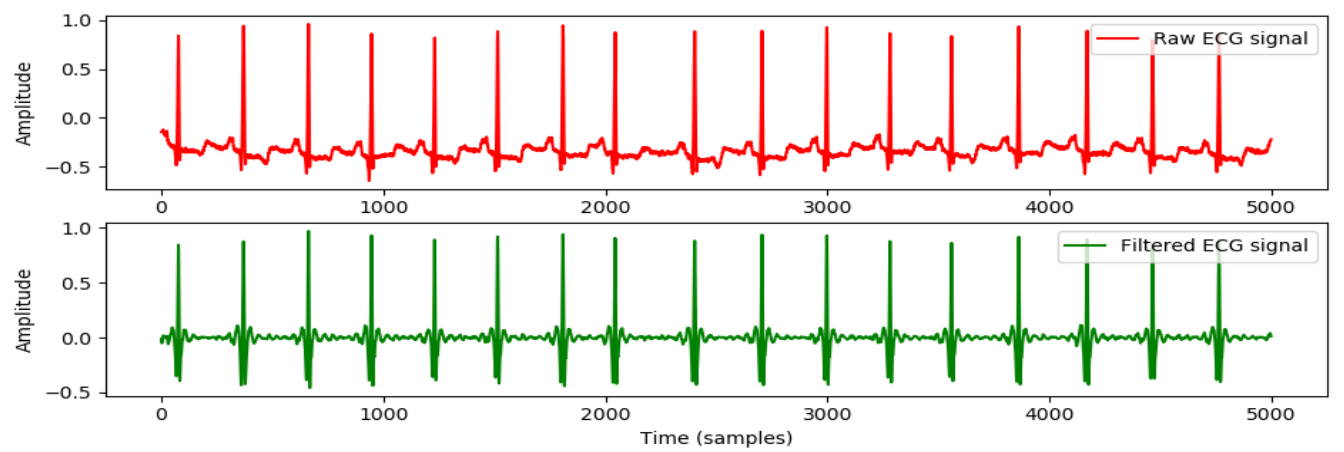

Fig. 1. The raw and filtered ECG segment of MIT-BIH's record 100.

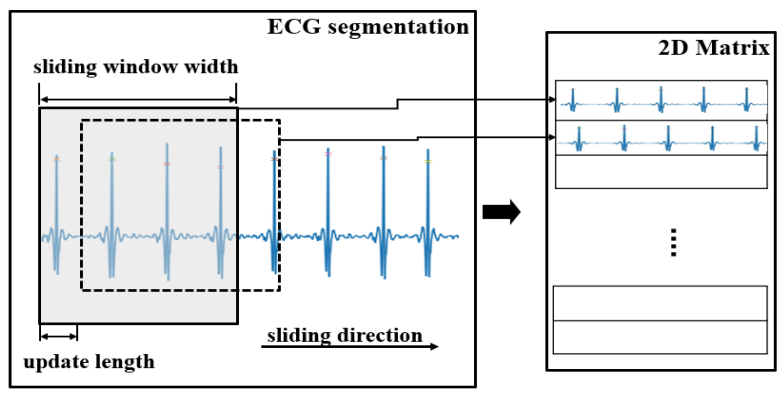

Fig. 2. Schematic illustration of the segmentation scheme.

3) Shuffle Scheme: For each patient, five subsets are defined by evenly dividing the above-mentioned ECG frames. Therefore, different subsets of different patients all containing a number of ECG frames ranging from 5173 to 5175, which is almost a 5-minute ECG recording. The purpose of this is to avoid the common problem in deep learning, overfitting, by shuffling the subset of different patients ECG records [16]. In addition, a subset of nearly 5 minutes in length will allow the LSTM to learn the hidden time relations contained therein. The details of the subset data shuffling scheme are shown in Fig. 3. The specific method is:

- Create five subsets for each patient's ECG recording.

- Create 90 numerically encoded ECG subsets from 18 recorded patients.

- Shuffle and combine the 90 ECG subsets into one ECG 2D matrix before the start of each training iteration.

- Utilize the reorganized ECG data as input of the proposed network.

By shuffling the ECG subsets, the proposed network is forced to learn more ECG data of different combinations, as these subsets are not only from different patients, but also in different positions in the patient's own complete ECG data. Furthermore, the contained frame is different in the time interval of the R-peak that will occur. This allows higher versatility of the proposed model and effectively prevents the overfitting problem from occurring.

\section{B. Confidence Label Computation}

Using the above segmented ECG and expert-labeled R-peak labels in the MIT-BIH arrhythmia database, a confidence label can be calculated to indicate the probability that $\mathrm{R}$ peaks will occur in the future. The ratio of the sampling point interval between the rightmost sampling point of the ECG frame and the next R peak (D) to the ECG frame length (L) is used to calculate the confidence label. Figure 4 gives the scheme for the proposed method. The probability of an R-peak occurring is determined as:

$$
C=1-\frac{D}{L}
$$

where

(D), represents the sampling point interval between the rightmost sampling point of the ECG frame and the next R peak; (L), represents the frame length of an ECG frame;

The greater the value of $\mathrm{C}$, the higher the probability of the occurrence of the R-peak. Therefore, when the value of $\mathrm{C}$ reaches the maximum value of 1 , the $\mathrm{R}$-peak occurs. This case happens when $\mathrm{D}$ is equal to 0 , that is, the rightmost sampling point of the ECG frame is exactly at the marked real R-peak, as shown in Fig. 4(b). Since the interval length between two adjacent R-peaks is about 500 sampling points, $\mathrm{C}$ is a nonnegative value. In addition, the range of $\mathrm{C}$ is affected by update size and frame length of the input ECG frame.

\section{Proposed CNN-LSTM Model}

1) Model structure: In this work, a novel method using ECG signals to predict future R-peak in real time is studied. Since the ECG signal is a chaotic time series, the proposed network should be able to capture the long-term dependence of data in the time dimension. The proposed network is a deep learning model that combines CNN and LSTM. Figure 5 illustrates the proposed architecture, and Table I gives a detailed overview of the structure.

The proposed model structure contains six 1D CNN layers, and every second 1D CNN layer is coupled with a max pooling layer to form a CNN set. ECG frames are directly fed into the 1D convolution layer to quickly extract low-dimensional sequential visual features and provide some noise filtering. The 


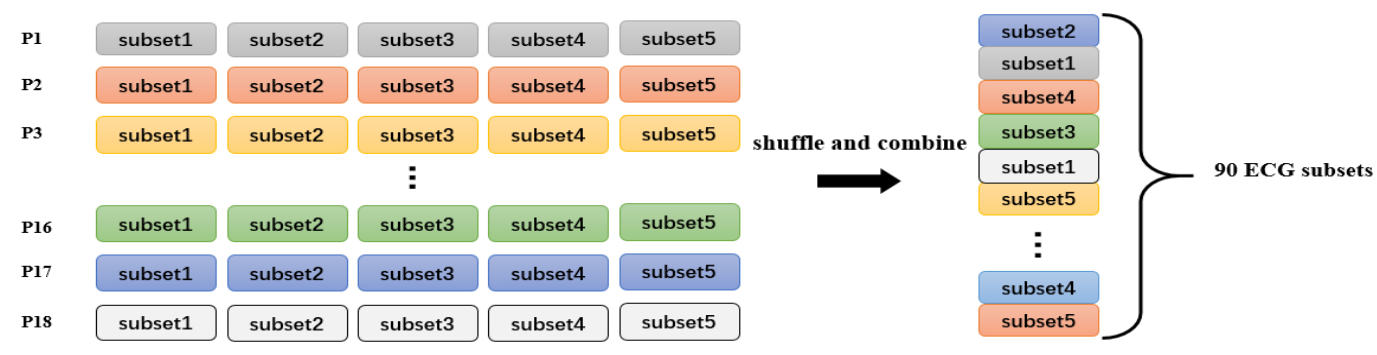

Fig. 3. Schematic illustration of the ECG subsets shuffle scheme.

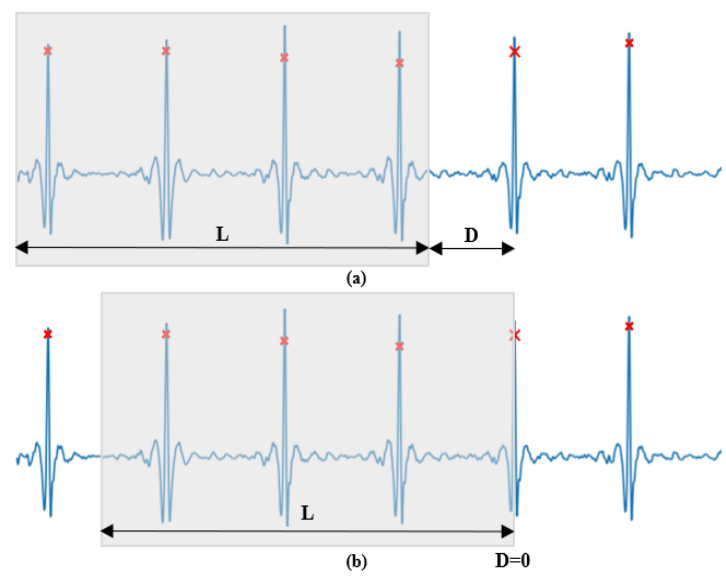

Fig. 4. An illustration of the proposed label computation method in two cases: the rightmost sampling point of the ECG frame is (a) not at the marked true R-peak (b) at the marked true R-peak.

TABLE I

THE DETAILS OF PROPOSED CNN-LSTM STRUCTURE.

\begin{tabular}{c|c|c|c|c}
\hline Layers & Layer Type & Number of neurons & CNN kernel size & stride \\
\hline \hline 1 & $1 D$ Convolution & 32 & 5 & - \\
\hline 2 & $1 D$ Convolution & 32 & 5 & - \\
\hline 3 & Max-Pooling & 32 & - & 3 \\
\hline 4 & $1 D$ Convolution & 64 & 5 & - \\
\hline 5 & $1 D$ Convolution & 64 & 5 & - \\
\hline 6 & Max-Pooling & 64 & - & 3 \\
\hline 7 & $1 D$ Convolution & 128 & 5 & - \\
\hline 8 & $1 D$ Convolution & 128 & 5 & - \\
\hline 9 & Max-Pooling & 128 & - & 3 \\
\hline 10 & LSTM & 128 & - & - \\
\hline 11 & Dense & 128 & - & - \\
\hline 12 & Dense & 128 & - & - \\
\hline 13 & Dense & 1 & - & - \\
\hline
\end{tabular}

stacked convolution layers continue to extract more abstract features from the previously extracted features. Connecting to the LSTM layer, helps the model capture time information [11]. Finally, only the output of the last step of the LSTM layer is input into a series of fully connected layers to predict the future R-peak.

a) CNN sets: The CNN architecture contains $3 \mathrm{CNN}$ sets. The convolution layer implements feature detection, and convolves across the input ECG frame to extract the feature

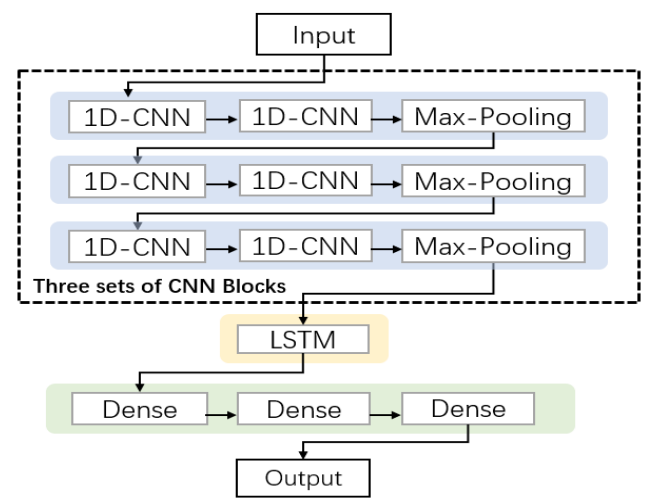

Fig. 5. The architecture of proposed CNN-LSTM mode.

map. Weights are adjusted after each convolution to produce the best function of the model. The feature extraction method of the 1-D convolution layer is shown in Fig. 6. Max-pooling layer takes the max value of multiple features to prevent overfitting and to reduce calculation costs. Since strides is set to 3 , the size of the output matrix is one third of the input, which reduces the complexity of the next layer.

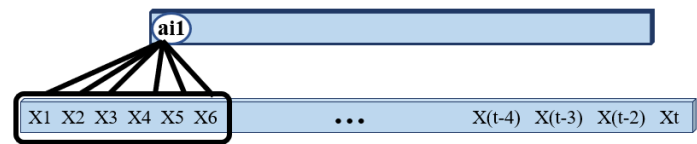

Fig. 6. Schematic diagram of 1D-CNN layer feature extraction.

b) Dense layer: The Dense layer realizes the full connection of the output of the last LSTM layer. Compared with directly using the LSTM layer for prediction, using the dense layer is easier to predict the output correctly as it has stronger discriminative ability [10]. Moreover, the dense layer and the connected LSTM layer are designed to have the same number of neurons to preserve the information between them [9].

c) Activity function: Relu is used as the activation function of the CNN layers and the fully connected layers. This is because our research problem is a regression problem implemented utilizing deep networks, and Relu always offsets the effect of the disappearance of the gradient in the deep network. The tanh activation function is used in the LSTM layer. The range of the tanh function is $\mathrm{r}[-1,1]$, which allows negative and positive feedback in the LSTM layer without 
exponential feedback, so that the LSTM layer can remember different past information.

d) Loss function: A loss function is used to measure the difference between the model's predict label and the true label, to indicate the direction for model optimization. In the experiment, we use Mean Squared Error (MSE) Loss function. MSE is the sum of squared differences between the predicted and the true labels, where $\mathrm{n}$ represents the number of labels. The formula is given by:

$$
\operatorname{MSE}(y, \hat{y})=\frac{1}{n} \sum_{i=1}^{n}\left(y_{i}-\hat{y}_{i}\right)^{2}
$$

The initial learning rate is set to 0.01 , and the 'Adam' algorithm is used to train the proposed model. We utilize a learning rate adjuster to adjust the learning rate based on the loss of the training set. If the value of loss cannot be reduced within five epochs, the learning rate will be reduced to $20 \%$ of the previous, until it drops to 0.00001 . In addition, the early stopping method is also utilized. If the accuracy is not improved within 20 epochs, the training operation is stopped.

\section{EXPERIMENTS AND RESULTS}

The method described in Section III was applied to the training data set. Using the model generated, effectiveness of the method was evaluated by considering the accuracy of Rpeak predictions from ECG signals of the test data set.

\section{A. Experimental setting}

1) Evaluation Metrics:

a) Root Mean Square Error (RMSE): Since the model is expected to achieve a R-peak forecast in real-time, with highprecision prediction of the $\mathrm{C}$ parameter, the model best fits a time series regression task and thus requires evaluation metrics for a regression problem. Root Mean Square Error (RMSE) was selected to generate the evaluation metrics of the model on the basis of its history as a widely successful evaluation tool for regression tasks. RMSE is given by the expression shown in (3).

$$
R M S E(y, \hat{y})=\sqrt{\frac{1}{n} \sum_{i=1}^{n}\left(y_{i}-\hat{y}_{i}\right)^{2}}
$$

The metrics popular use in literature facilitates comparison between a great number of models previously produced. Additionally, it allows comparative analysis of future networks with relative ease given the use of RMSE, throughout.

b) Custom Accuracy Metric: A custom accuracy metric that considers the tolerance range is proposed as an additional metric for the evaluation of the model. The tolerance range is defined as the window that determines the accuracy of a prediction given the forecast difference. Specifically, when the difference between the predict label and the calculated label is within the tolerance range, the value is treated as a correct prediction.

The model utilises it a custom metric defined as the confidence label $(\mathrm{C})$, which is calculated from the true labels $(y)$ to give the ground truth values such that the predicted values can be compared. However, this means that the predicted values $(\hat{y})$ are received from the model as confidence label (C) metrics and therefore must be converted to the time-domain such that the R-peak value can be accurately derived as a valid time index. The expression described in (4) outlines the conversion between the two parameters.

$$
\begin{array}{rlr}
\hat{C} & = & 1-\frac{D \pm S}{L} \\
& = & \frac{L}{L}-\left(\frac{D \pm S}{L}\right) \\
& = & \frac{L-D}{L}+\frac{D \mp S}{L} \\
& = & \left(1-\frac{D}{L}\right)+\frac{\mp S}{L} \\
& = & C+\frac{\mp S}{L} \\
& = & C+(\mp R)
\end{array}
$$

where

(S) represents the sampling point interval between the predicted label and the true label; and

(R) represents the custom confidence label-based tolerance range of the prediction.

Thus, the prediction error expressed in sampling point interval (S) is determined by the ECG frame length (L) and confidence label-based tolerance range $(R)$ given by,

$$
S=R L
$$

The sampling frequency $f_{s}$ of the ECG database used is 360 hertz. According to (6), the duration of each sampling point is calculated to be about 0.002788 seconds.

$$
T=\frac{1}{f_{s}}
$$

Therefore, based on the derivation in (5), the time-based tolerance range $(\mathrm{M})$, expressed in seconds, of the prediction is derived as:

$$
M=S T=R L T
$$

In this paper, according to ANSI/AAMI EC38 and EC57 standards [15], the range of this window is set to \pm 75 milliseconds, which corresponds to \pm 25 sampling points around the real label position and a range of \pm 0.025 for the custom confidence label.

\section{B. Results}

Training of the model utilized 80 complete epochs and the network structure proposed by section III-C. The model was then evaluated on the test set, which included three randomly selected records, 112,122, and 117, each of which were not included in the training data set. Testing ECG signals were prepared using the same methods as done for training data set, including noise reduction, segmentation, and confidence label computation. 
TABLE II

TEST ACCURACY AND RMSE FOR EACH RECORD

\begin{tabular}{|c|c|c|c|c|}
\hline & 112 & 122 & 117 & Overall Average \\
\hline \hline RMSE & 0.0171 & 0.0099 & 0.0464 & 0.0291 \\
\hline Accuracy $(\%)$ & 94.28 & 98.81 & $\mathbf{7 8 . 9 9}$ & 90.68 \\
\hline
\end{tabular}

The RMSE and accuracy of the resulting R-peak predictions for each record and their overall average are summarized in Table II.

A demonstration of the predictions found by the proposed method is shown in Fig.7. Here, an ECG segment containing 3000 sample points was selected from each of the three records of the test set, and predictions were found. The red cross on the figure represents the real label marked by experts, while the green cross represents that prediction of the model. It is worth
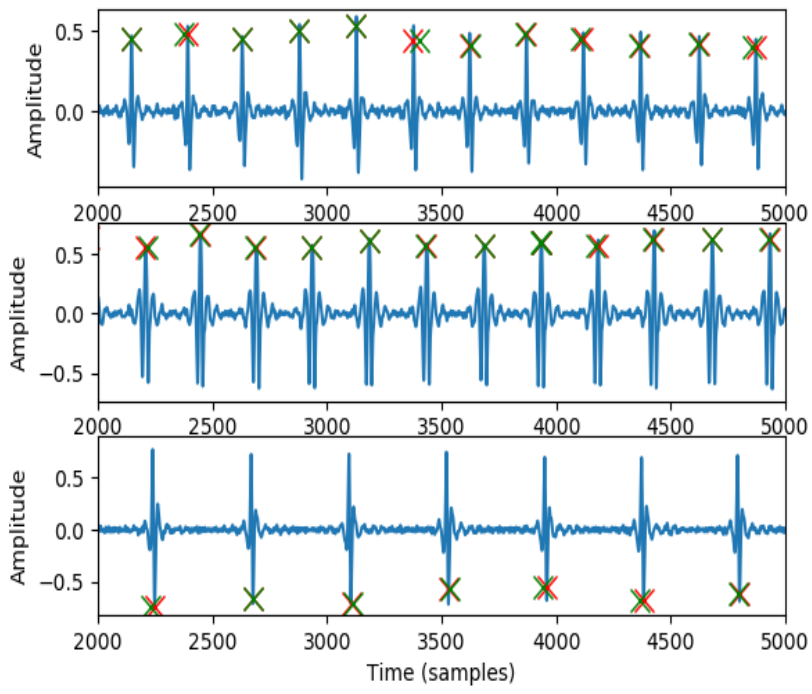

Fig. 7. ECG signals showing R-peaks predicted by the model. The red cross on the figure represents the real label marked by experts, while the green cross represents that prediction of the model.

mentioning that the accuracy of record 117 is significantly lower than the other two records. This is because some ECG records in the MIT-BIH database have annotations where the beat label is not placed on the R-peak, but on the QRS complex more generally. As our proposed method requires accurate Rpeak annotations for training and valid accuracy testing, future work will further evaluate the method with a data set with more accurate R-peak annotations.

Furthermore, although the number of ECG frames for model training is increased through overlapping, only 18 records are currently used. Future work will use more training data to improve the performance of deep learning models.

\section{CONCLUSION AND FUtURE WORK}

The proposed real-time $\mathrm{R}$ peak prediction method has many advantages. It realizes the prediction of R-peak before the ECG signal is recorded, which is a novel idea. Results presented here are from preliminary work in this area. However, the results obtained indicate that this method has considerable possibilities for further improvement and is worthy of in-depth study. Future research will evaluate the impact of the network structure and hyperparameters used to generate the model, as well as the accuracy of annotations of the data set used for training.

\section{REFERENCES}

[1] W. H. Organization et al., "Health topics: Cardiovascular diseases," See http://www. who. int/topics/cardiovascular_diseases/en//last checked July 2012), 2013.

[2] S. M. P. Dinakarrao, A. Jantsch, and M. Shafique, "Computer-aided arrhythmia diagnosis with bio-signal processing: A survey of trends and techniques," ACM Computing Surveys (CSUR), vol. 52, no. 2, pp. 1-37, 2019.

[3] F. Shaffer, R. McCraty, and C. L. Zerr, "A healthy heart is not a metronome: an integrative review of the heart's anatomy and heart rate variability," Frontiers in psychology, vol. 5, p. 1040, 2014.

[4] S. Hong, Y. Zhou, J. Shang, C. Xiao, and J. Sun, "Opportunities and challenges of deep learning methods for electrocardiogram data: A systematic review," Computers in Biology and Medicine, p. 103801, 2020.

[5] Y. Xiang, Z. Lin, and J. Meng, "Automatic qrs complex detection using two-level convolutional neural network," Biomedical engineering online, vol. 17, no. 1, p. 13, 2018.

[6] U. R. Acharya, H. Fujita, S. L. Oh, Y. Hagiwara, J. H. Tan, and M. Adam, "Application of deep convolutional neural network for automated detection of myocardial infarction using ecg signals," Information Sciences, vol. 415, pp. 190-198, 2017.

[7] Y. Qian, M. Bi, T. Tan, and K. Yu, "Very deep convolutional neural networks for noise robust speech recognition," IEEE/ACM Transactions on Audio, Speech, and Language Processing, vol. 24, no. 12, pp. 22632276, 2016.

[8] S. Hochreiter and J. Schmidhuber, "Long short-term memory," Neural computation, vol. 9, no. 8, pp. 1735-1780, 1997.

[9] B. Yuen, X. Dong, and T. Lu, "Inter-patient cnn-lstm for qrs complex detection in noisy ecg signals," IEEE Access, vol. 7, pp. 169359-169370, 2019.

[10] T. N. Sainath, O. Vinyals, A. Senior, and H. Sak, "Convolutional, long short-term memory, fully connected deep neural networks," in 2015 IEEE International Conference on Acoustics, Speech and Signal Processing (ICASSP), pp. 4580-4584, IEEE, 2015.

[11] S. L. Oh, E. Y. Ng, R. San Tan, and U. R. Acharya, "Automated diagnosis of arrhythmia using combination of cnn and 1stm techniques with variable length heart beats," Computers in biology and medicine, vol. 102, pp. 278-287, 2018.

[12] J. H. Tan, Y. Hagiwara, W. Pang, I. Lim, S. L. Oh, M. Adam, R. San Tan, M. Chen, and U. R. Acharya, "Application of stacked convolutional and long short-term memory network for accurate identification of cad ecg signals," Computers in biology and medicine, vol. 94, pp. 19-26, 2018.

[13] U. R. Acharya, S. L. Oh, Y. Hagiwara, J. H. Tan, M. Adam, A. Gertych, and R. San Tan, "A deep convolutional neural network model to classify heartbeats," Computers in biology and medicine, vol. 89, pp. 389-396, 2017.

[14] G. B. Moody and R. G. Mark, "The impact of the mit-bih arrhythmia database," IEEE Engineering in Medicine and Biology Magazine, vol. 20, no. 3, pp. 45-50, 2001.

[15] A. ECAR, "Recommended practice for testing and reporting performance results of ventricular arrhythmia detection algorithms," Association for the Advancement of Medical Instrumentation, vol. 69, 1987.

[16] K. M. Tsiouris, V. C. Pezoulas, M. Zervakis, S. Konitsiotis, D. D Koutsouris, and D. I. Fotiadis, "A long short-term memory deep learning network for the prediction of epileptic seizures using eeg signals," Computers in biology and medicine, vol. 99, pp. 24-37, 2018. 